\title{
A patient with extensive meningeal calcification due to pseudohypoparathyroidism: A case report
}

\author{
Mahmoud Salih Daoud ${ }^{1}$, Abubakr Abdulla Elamin ${ }^{1}$, Ahmed Rizgalla Mohammed ${ }^{1}$, Ala \\ Khalid $^{1}$, Abdallah Hassan ${ }^{1}$, and Arif Taha ${ }^{1}$ \\ ${ }^{1}$ University of Gezira Faculty of Medicine
}

March 4, 2022

\begin{abstract}
A 35-year-old man with 18-year history of epilepsy on Carbamazepine, was diagnosed with hydrocephalus complicated by secondary severe optic atrophy and underwent Ventriculo-Peritoneal shunt. Imaging revealed Falx Cerebri, Supratentorial calcification, biochemical tests showed hypocalcemia, hyperphosphatemia and elevated parathyroid hormone. Patient was given Vitamin D and Calcium and his seizures resolved.
\end{abstract}

\section{A patient with extensive meningeal calcification due to pseudohypoparathyroidism:} a case report.

\section{Abstract}

A 35-year-old man with 18-year history of epilepsy on Carbamazepine, was diagnosed with hydrocephalus complicated by secondary severe optic atrophy and underwent Ventriculo-Peritoneal shunt. Imaging revealed Falx Cerebri, Supratentorial calcification, biochemical tests showed hypocalcemia, hyperphosphatemia and elevated parathyroid hormone. Patient was given Vitamin D and Calcium and his seizures resolved.

Keywords : Pseudohypoparathyroidism, Meningeal calcification, Hydrocephalus.

\section{Key Clinical Message}

In spite of PHP is a very rare disorder of young, yet it has to be considered in cerebral and meningeal calcification, even in old patients.

\section{Introduction}

Pseudohypoparathyroidism is a very rare disorder, with estimated prevalence between 0.3 and 1.1 cases per 100000 populations, only 60 cases have been reported worldwide as of December $2016^{1}$.

Pseudohypoparathyroidism is defined as a heterogeneous group of rare endocrine diseases characterized by normal renal function with resistance to parathyroid hormone functions, manifesting with hypocalcemia, hyperphosphatemia, and high levels of concentration in serum parathyroid hormone $(\mathrm{PTH})^{2}$. Diagnosis of pseudohypoparathyroidism (PHP) is mainly biochemical, confirmed by coexistence of hypocalcemia and hyperphosphatemia with elevated PTH levels in the presence of normal vitamin D values, normal renal function and the absence of hypercalciuria.

PHP can be additionally (confirmed) diagnosed by genetic testing for a mutation in the GNAS1 gene and identification of subtypes. These subtypes are divided based on several phenotypes: 
Type Ia - caused by mutations in GNAS - and Type Ic: normal Gs-alpha activity together with the absence of GNAS mutations. They have been associated with Albright hereditary osteodystrophy, decreased response to exogenous PTH as measured by urine cyclic adenosine monophosphate (cAMP) and urine phosphorus, low serum calcium, generalized endocrine resistance (affecting any hormone that relies on Gs-alpha signaling), and autosomal dominant inheritance.

Type Ib is caused by mutations in GNAS.

In Type II, the underlying genetic mutation is unknown, characterized by no association to skeletal phenotype, decreased response to exogenous PTH as measured by urine cAMP and urine phosphorus, low serum Calcium, and hormonal resistance limited to PTH target tissue; however, there are an increasing number of cases associated with thyroid-stimulating hormone resistance and inheritance is sporadic ${ }^{3}$.

The aim of this case report is to describe the clinical diversity of PHP and to emphasize the importance of the basic approach using laboratory tests in patients with symptomatic hypocalcemia. A calcium-phosphorus profile properly interpreted should lead to an accurate diagnosis and timely treatment of PHP. This report, besides being the first case report from Sudan as a lower-income country with limited facilities, our patient had an unusual presentation of meningeal calcification at late adulthood. This brings novelty to our study because we suggest the possibility of shortcomings in the diagnostic approach to patients with hypocalcemia. Perhaps calcium disorders are underdiagnosed and sometimes overlooked as a differential diagnosis of frequent clinical expressions, such as seizures and other neuromuscular symptoms.

\section{Case presentation :}

A 35-year-old man, with a 18-year history of epilepsy, on Carbamazepine $400 \mathrm{mg}$ once/daily, with a rate of 4 seizures per year. The patient was referred from the neurosurgical department for controlling his seizures. He was diagnosed with hydrocephalus complicated by severe optic atrophy and underwent insertion of a ventriculoperitoneal shunt. Prior brain imaging (CT/MRI) ( images 1.2.3.4) revealed the existence of hydrocephalus and meningeal calcification. The patient had no other symptoms suggesting motor, sensory, cerebellar, sphincter and autonomic abnormalities. There was no past history of head trauma, childhood cerebral infection or skin tumors. He is neither diabetic nor hypertensive, and has a negative family history for both conditions.

On examination, the patient was $70 \mathrm{~kg}$ in weight and $160 \mathrm{~cm}$ in height. Neurological examinations demonstrated a Glasgow Coma Scale (GCS) of 15 and orientation to time, place and person, normal behavioral and cognitive function with no apparent dysmorphological or congenital anomalies. Cranial nerves examination showed optic nerve abnormalities, a significant decrease in visual acuity (legally blind) with evidence of secondary severe optic disc atrophy and papilledema on Fundoscopic examination.

Other cranial nerves were normal, with no acute abnormalities of motor, sensory, cerebellar and sphincteric function. Additionally, there were positive Chvostek and Trousseau signs. Otherwise systemic examination was all clear.

Biochemical blood tests (table 1) showed low serum Calcium $(6.8 \mathrm{mg} / \mathrm{dl})$, normal vitamin $\mathrm{D}$, urea and Creatinine. Elevated serum phosphate $(6.2 \mathrm{mg} / \mathrm{dl})$ and elevated parathyroid hormone $(118 \mathrm{pg} / \mathrm{ml})$ were present.

Optical coherence tomography for retina (OCT) revealed optic disc edema. Fundus photos showed secondary severe optic atrophy and moderate optic disc edema.

Full body X-rays, echocardiography and ECG were normal.

The patient was given supplemental Vitamin D and Calcium oral tablets for three months; subsequently all hypocalcemic signs have improved, and seizures have subsided since.

He is scheduled for routine follow-up through monitoring of serum calcium, phosphate, RFT, Vitamin D for 3 months, followed by one year, then every 6 months for life, with annual brain imaging. 


\section{Discussion :}

Our patient, a middle-aged man with a 17-year history of seizures presented with hydrocephalus and extensive meningeal calcification. Initially, falx cerebri calcification was misleading us toward nevoid basal cell carcinoma syndrome as it presentes similarly, although the absence of major and minor criteria excluded it as a diagnosis ${ }^{4}$.

Brain calcification was usually investigated in PHP due to the hyperphosphatemia.

In most conditions, seizures and epilepsy are the common clinical manifestations resulting from the calcification of subcortical region and basal ganglia, some patients present with cerebral and cerebellar calcification as well as subcutaneous calcifications ${ }^{5}$.

However, few cases were ever reported with these manifestations, yet no other cases similar to our case of falx cerebri exist in published literature and supratentorial calcification along with secondary hydrocephalus is an uncommon presentation for this syndrome.

On the other hand, the presence of low serum calcium, high phosphate, and high parathyroid hormone despite normal RFT and vitamin D levels led us to consider pseudohypoparathyroidism as a diagnosis.

In accordance with the recommendations of the recently published first consensus statement on the diagnosis and management of pseudohypoparathyroidism-related disorders, the diagnosis should be based on clinical and biochemical characteristics, which will vary according to the age of the patient ${ }^{6}$.

Absence of skeletal deformities and family history confirmed that this patient has either type Ib or type II which are sporadic disorders; unfortunately due to facility limitations in Sudan, confirming the diagnosis with genetic phenotyping and mapping was difficult ${ }^{7}$.

Although PHP is most commonly diagnosed during childhood or adolescence, one case from Italy illustrated the history of a patient of pseudohypoparathyroidism type 1A, which went undiagnosed until her 60s, with multi-hormonal resistance and clinical complications throughout her life ${ }^{6}$. Our case phenomenally describes late adulthood diagnosis of the condition, thus highlighting the importance of diagnosing this rare disease, which had a great impact on patients and their family life as this is crucial to the appropriate treatment and lifelong management of the disease and its complications. However, late diagnosis led to development of complications such as hydrocephalus and blindness which affected the social life of the patient

Starting the calcium and vitamin D supplemental treatment promptly corrected the patient's hypocalcemia, and stopped both muscle spasms and seizures as hypocalcemia and the development of meningeal calcifications could have contributed to the development of these seizures ${ }^{8}$.

\section{Conclusion}

Despite PHP being a rare possibility, it should be considered in the diagnostic algorithm (figure 1) of hypocalcemia, especially when there is epilepsy or dural calcifications, even in elderly patients. The present case underlines the importance of early diagnosis of pseudohypoparathyroidism, as this is crucial to the appropriate treatment and lifelong management of the disease and its complications, which have a great impact on patients and their families.

\section{Strengths}

Prompt diagnosis and management hypocalcemia of pseudohypoparathyroidism led to an excellent outcome in this patient.

\section{Limitations}

The GNAS mutation and the urinary cAMP response to exogenous PTH were not performed due to limited resources in Sudan.

Abbreviations : PHP:pseudohypoparathyroidism, PTH: Parathyroid hormone, GSC: Glasgow Coma Scale. 


\section{References:}

1. Ucciferro P, Anastasopoulou C. Pseudohypoparathyroidism. [Updated 2021 Jul 21]. In: StatPearls [Internet]. Treasure Island (FL): StatPearls Publishing; 2021 Jan-. Available from: https://www.ncbi.nlm.nih.gov/books/NBK547709/

2. Mantovani G, Linglart A, Garin I, Silve C, Elli FM, de Nanclares GP. Clinical utility gene card for: pseudohypoparathyroidism. Eur J Hum Genet. 2013 Jun. 21 (6):[Medline]. [Full Text].

3. Hendy GN, Cole DEC, Bastepe M. Hypoparathyroidism and Pseudohypoparathyroidism. https://www.ncbi.nlm.nih.gov/books/NBK279165/\#!po=0.216450

4. Veenstra-Knol HE, Scheewe JH, van der Vlist GJ, van Doorn ME, Ausems MG. Early recognition of basal cell naevus syndrome. Eur J Pediatr. 2005 Mar;164(3):126-30. doi: 10.1007/s00431-004-1597-4. Epub 2004 Dec 10. PMID: 15717176.

5. Evans BK, Donley DK. Pseudohypoparathyroidism, Parkinsonism syndrome, with no basal ganglia calcification. J Neurol Neurosurg Psychiatry 1988;51:709-13 Song et al. Medicine (2017) 96:11

6. Patrizia Del Monte, Carla Micaela Cuttica, Alessandro Marugo, Luca Foppiani, Daniela Audenino, Tomasz Tadeusz Godowicz, Francesca Marta Elli, Giovanna Mantovani, Emilio Di Maria, "Unrecognized Pseudohypoparathyroidism Type 1A as a Cause of Hypocalcemia and Seizures in a 64-Year-Old Woman", Case Reports in Endocrinology, 2019, 8456239. https://doi.org/10.1155/2019/8456239

7. G. Mantovani, M. Bastepe, D. Monk et al., "Diagnosis and management of pseudohypoparathyroidism and related disorders: first international Consensus Statement," Nature Reviews Endocrinology, 2018;14(8):476-500. https://doi.org/10.1155/2019/8456239

8. G. Mantovani, M. Bastepe, D. Monk et al., "Diagnosis and management of pseudohypoparathyroidism and related disorders: first international Consensus Statement," Nature Reviews Endocrinology, vol. 14, no. 8, pp. 476-500, 2018

\section{Hosted file}

images.docx available at https://authorea.com/users/463509/articles/558580-a-patient-withextensive-meningeal-calcification-due-to-pseudohypoparathyroidism-a-case-report

\section{Hosted file}

Table and figure.docx available at https://authorea.com/users/463509/articles/558580-apatient-with-extensive-meningeal-calcification-due-to-pseudohypoparathyroidism-a-casereport 